\title{
Characterization of fluorescent NBD-cholesterol efflux in THP-1-derived macrophages
}

\author{
WEI SONG ${ }^{*}$, WEI WANG ${ }^{*}$, YU WANG, LIYANG DOU, LIANFENG CHEN and XIAOWEI YAN \\ Department of Cardiology, Peking Union Medical College Hospital, \\ Chinese Academy of Medical Sciences and Peking Union Medical College, Beijing 100730, P.R. China
}

Received September 9, 2014; Accepted June 26, 2015

DOI: $10.3892 / \mathrm{mmr} .2015 .4154$

\begin{abstract}
Macrophage cholesterol efflux is important in maintaining cellular lipid homeostasis and preventing the formation of lipid-laden foam cells. Although radioactive $\left[{ }^{3} \mathrm{H}\right]$-cholesterol is widely used as a tracer in cholesterol efflux assays, the lengthy and labor-intensive assay procedure, and the radioactivity disposal procedure limit the use of this assay for high-throughput screening. In the present study, a novel procedure using fluorescent N-(7-nitrobenz-2-oxa-1,3-diazol-4-yl) amino)-23,24-bisnor-5-xholen-3 $\beta$-ol (NBD)-cholesterol was developed as a substitute for $\left[{ }^{3} \mathrm{H}\right]$-cholesterol for the measurement of cholesterol efflux in THP-1-derived macrophages. NBD-cholesterol uptake and metabolism in the THP-1 cells were characterized using fluorescent microscopy and spectrophotometry. NBD-cholesterol distributed rapidly into the cell organelles, with the exception of the nucleus. The uptake of NBD-cholesterol in the THP-1 macrophages was concentration- and time-dependent, and reached a plateau following $4 \mathrm{~h}$ incubation. The present study subsequently
\end{abstract}

Correspondence to: Dr Xiaowei Yan, Department of Cardiology, Peking Union Medical College Hospital, Chinese Academy of Medical Sciences and Peking Union Medical College, 1 Shuaifuyuan, Beijing 100730, P.R. China

E-mail: xiaoweiyanpumc@yahoo.com.cn

${ }^{*}$ Contributed equally

Abbreviations: 22-NBD-cholesterol, 22-(7-nitrobenz-2-oxa-1, 3-diazol-4-yl-amino)-23, 24-bisnor-5-cholen-3 $\beta$-ol; PMA, phorbol myristate acetate; FBS, fetal bovine serum; RCT, reverse cholesterol transport; PBMCs, human peripheral blood mononuclear cells; BODIPY, boron-dipyrromethene; DHE, dehydroergosterol; apoA-1, apolipoprotein A-1; HDL, high-density lipoprotein; Ox-LDL, oxidized low-density lipoprotein; $\left[{ }^{3} \mathrm{H}\right]$-cholesterol, tritium-labeled cholesterol; ABCA1, ATP binding cassette transporter A1; ABCG1, ATP binding cassette transporter G1; SR-B1, scavenger receptor class B type I

Key words: N-(7-nitrobenz-2-oxa-1,3-diazol-4-yl)amino)-23,24bisnor-5-xholen-3 $\beta$-ol-cholesterol, cholesterol efflux, macrophage, atherosclerosis, high-density lipoprotein, apolipoprotein A-1 investigated whether NBD-cholesterol efflux was correlated with $\left[{ }^{3} \mathrm{H}\right]$-cholesterol efflux in THP-1 derived macrophages and in human peripheral blood mononuclear cells (PBMCs). The results demonstrated that the percentage of efflux of NBD-cholesterol in the THP-1 cells was significantly correlated with that of $\left[{ }^{3} \mathrm{H}\right]$-cholesterol, at various concentrations of HDL or apoA- 1 as lipid acceptors $\left(\mathrm{R}^{2}=0.876\right.$ for $\mathrm{HDL} ; \mathrm{R}^{2}=0.837$ for apoA-1; $\mathrm{P}<0.001)$. In the PBMCs, NBD-cholesterol efflux also correlated significantly with $\left[{ }^{3} \mathrm{H}\right]$-cholesterol efflux $\left(\mathrm{R}^{2}=0.887\right.$ for HDL; $\mathrm{R}^{2}=0.872$ for apoA-1; $\left.\mathrm{P}<0.001\right)$. Furthermore, NBD-cholesterol efflux in the THP-1 cells exhibited a similar trend to that obseved in the PBMCs. In conclusion, the results of the present study suggested that fluorescent NBD-cholesterol can be used as a sensitive and specific probe in cholesterol efflux assays in THP-1-derived macrophages.

\section{Introduction}

Coronary artery disease (CAD) is the leading contributor to morbidity and mortality rates worldwide, and results in a mortality rate of $>7,000,000$ per year (1). Atherosclerotic plaques are key in the pathophysiology of CAD, in which macrophages initially become foam cells and initiate plaque formation. The imbalance of cholesterol uptake and efflux in macrophages may cause excessive accumulation of cholesterol and foam cell formation, which is a hallmark in the development of atherosclerosis (2). Cholesterol efflux from macrophages is a critical step in reverse cholesterol transport, which may prevent macrophages from becoming foam cells and thus reduce atherosclerosis $(3,4)$. Free cholesterol is transported from macrophages in the arterial atherosclerotic plaque to an extracellular acceptors, including apoliporotein (apo) A-1 and high-density lipoprotein (HDL), and then back to the liver for bile acid synthesis and excretion (5). Previously, Khera et al reported a reverse correlation between cholesterol efflux capacity and atherosclerosis (6). To understand the underlying mechanism regulating cholesterol efflux, it is important to develop a reliable assay for measuring cholesterol efflux in vitro.

Traditionally, radioisotope-labeled cholesterols have been used as probes in cholesterol efflux assays. However, the procedure is lengthy and labor-intensive, and the radioactivity disposal procedure can limit the use of this assay in high-throughput screening. By contrast, fluorescent 
cholesterol analogs have demonstrated potential as suitable alternatives for radioisotope-labeled cholesterols (7). For decades, fluorescent cholesterol analogs have been used in membrane biophysics for the investigation of lipid trafficking and the membrane organization of native cholesterol $(8,9)$. Until recently, fluorescent sterols were also used in cholesterol efflux assays as an alternative for radioisotope-labeled cholesterol $(10,11)$. Commonly used fluorescent analogs of cholesterol include N-(7-nitrobenz-2-oxa-1,3-diazol-4-yl) amino)-23,24-bisnor-5-xholen-3 $\beta$-ol (NBD)-cholesterol, boron-dipyrromethene (BODIPY)-cholesterol and dehydroergosterol with intrinsic fluorescence (8). The analogs bearing the NBD fluorophore have demonstrated advantageous properties for membrane investigations. There are three versions of NBD-cholesterol: The NBD flurophore attached to the $3 \beta-\mathrm{OH}$ group of cholesterol, NBD linked to carbon 22 in the sterol side chain (22-NBD-cholesterol) and NBD linked to carbon 25 (25-NBD-cholesterol).

The THP-1 human monocytic leukemia cell line is a differentiated monocytic cell line, which exhibites macrophage-like characteristics when stimulated with phorbol ester $(12,13)$ Notably, THP-1-derived macrophages have been widely used as a suitable in vitro model for investigating cholesterol efflux (14-16). At present, there is a lack of information regarding the use of fluorescent cholesterol analogs, including NBD-cholesterol, in efflux assays in THP-1-derived macrophages. The present study aimed to characterize a cholesterol efflux assay using fluorescent NBD-cholesterol as a probe in THP-1-derived macrophages, as well as in human peripheral blood mononuclear cells (PBMCs). The results may assist in the development of an in vitro model for cholesterol efflux, which can be used as a high-throughput screening assay.

\section{Materials and methods}

Reagents and materials. THP-1 cells were obtained from the Cell Bank of Basic Research Institute of the Chinese Academy of Medicine Sciences (Beijing, China). RPMI 1640 medium and Triton X-100 were purchased from Gibco Life Technologies (Carlsbad, CA, USA). Phorbol myristate acetate (PMA) and macrophage colony stimulating factor (M-CSF) were purchased from Sigma-Aldrich (St. Louis, MO, USA). 22-(N-nitrobenz-2-oxa-1,3-diazol-4-yl-amino)-23, 24-bisnor-5-cholen-3-ol (NBD)-cholesterol was obtained from Molecular Probes (Eugene, OR, USA). The fluorescent cholesterol was diluted in ethanol, with a final concentration of $0.5 \%$ ethanol. Purified human high-density lipoprotein (HDL) and apolipoprotein A1 (apoA-1) were obtained from CalBiochem (San Diego, CA, USA). Ficoll-Paque Premium was purchased from Haoyang Biosciences (Tianjing, China). Ox-LDL was purchased from the Basic Research Institute of the Chinese Academy of Medicine Sciences (cells were preloaded with $50 \mu \mathrm{g} / \mathrm{ml}$ Ox-LDL for $48 \mathrm{~h}$ ).

Isolation of human PBMCs. The human PBMCs were isolated from 13 volunteers. All the volunteers were healthy men (average age, 32 years) screened in Peking Union Medical College Hospital (Beijing, China). A total of $30 \mathrm{ml}$ of blood was collected by venipuncture following $12 \mathrm{~h}$ fasting, which was anticoagulated with heparin vacutainer tubes (BD
Biosciences, San Jose, CA, USA). The procedures of the present study were approved by the Human Ethics Committee of Peking Union Medical College Hospital (Beijing, China; registration no. ChiCTR-RCH-10000748). Each participant provided written informed consent.

The isolation of PBMCs was performed by gradient centrifugation at room temperature for $40 \mathrm{~min}$ at $400 \mathrm{x} \mathrm{g}$, by layering $30 \mathrm{ml}$ heparinized $(10 \mathrm{U} / \mathrm{ml})$ blood over $15 \mathrm{ml}$ NycoPrep (Invitrogen Life Technologies, Carlsbad, CA, USA). Following centrifugation at room temperature for $40 \mathrm{~min}$ at $400 \mathrm{x} \mathrm{g}$ on a Ficoll-Paque density gradient, the resting human PBMCs were collected from the interface and washed with $0.9 \%(\mathrm{w} / \mathrm{v})$ sodium chloride (17). The viability and purity of the monocytes were determined using flow cytometric analysis (CD14 staining), which confirmed a purity of at least $85 \%$ in all the experiments. The culture conditions were set at $37^{\circ} \mathrm{C}$ in a humidified atmosphere with $5 \% \mathrm{CO}_{2}$. The cells were cultured in a polystyrene 12 -well plate at a density of $5 \times 10^{6}$ cells/ml in serum-free RPMI 1640 medium containing $25 \mathrm{mmol} / 1 \mathrm{HEPES}$ (Sigma-Aldrich) and $10 \mathrm{ng} / \mathrm{ml}$ human M-CSF for 4-6 h. Subsequently, the cells were cultured in RPMI 1640 containing 10\% autologous serum for 7 days. The macrophages were serum-starved for $6 \mathrm{~h}$ and washed with phosphate-buferred saline (PBS) prior to incubation with NBD-cholesterol.

Cell culture and treatment. The cells of the THP-1 human monocytic cell line were cultured in RPMI 1640 medium supplemented with $20 \%$ heat-inactivated fetal bovine serum (FBS) and $100 \mu \mathrm{g} / \mathrm{ml}$ penicillin-streptomycin in $5 \% \mathrm{CO}_{2}$ at $37^{\circ} \mathrm{C}$. The THP- 1 cells were seeded at a density of $2 \times 10^{6}$ in a 12-well plate (Costar; Corning Incorporated, New York, NY, USA) and differentiated into macrophages by treatment with $100 \mathrm{ng} / \mathrm{ml}$ PMA for $72 \mathrm{~h}$ (18). To assess NBD-cholesterol uptake, the differentiated THP-1 cells were incubated at $37^{\circ} \mathrm{C}$ with various concentrations of NBD-cholesterol $(0.1,1,5$ and $10 \mu \mathrm{mol} / \mathrm{l}$ ) in basic phenol red-free RPMI 1640 medium. Following incubation, the supernatant was removed and the cells were washed three times with PBS. Cellular cholesterol was then extracted using $0.1 \%$ Triton X-100. The cells were harvested at different time-points for the detection of fluorescence intensity (FI), and images were captured using a fluorescence microscope (EVOS-fl; Thermo Fisher Scientific, Waltham, MA, USA). Cellular NBD-cholesterol uptake was also evaluated under microscopy.

FI detection. The FI of NBD-cholesterol in the medium and cell lysate was measured in a black polystyrene 96 -well plate (Costar; Corning Incorporated) using a microplate spectrophotometer (EnVision ${ }^{\circledR}$; PerkinElmer, Inc., Waltham, MA, USA) at a wavelength of $469 \mathrm{~nm}$ for excitation and a wavelength of $537 \mathrm{~nm}$ for emission. The parameter of sensitivity was set to 100 and the final volume for the assay was $200 \mu \mathrm{l}$.

Cholesterol efflux assays. For the $\left[{ }^{3} \mathrm{H}\right]$-cholesterol assay, the cells were washed with cold PBS three times and were then labeled with $1 \mu \mathrm{Ci} / \mathrm{ml}\left[{ }^{3} \mathrm{H}\right]$-cholesterol (NEN Life Science Products, Inc., Boston, MA, USA) in 10\% FBS RPMI 1640 medium for $12 \mathrm{~h}$. Following incubation at $37^{\circ} \mathrm{C}$, the cells were washed with PBS and equilibrated for an additional $2 \mathrm{~h}$ at $37^{\circ} \mathrm{C}$ 
in medium containing 2\% FBS, apoA-1 or HDL, used as a lipid acceptor, in which the cells were incubated for an additional $4 \mathrm{~h}$ at $37^{\circ} \mathrm{C}$. The same concentrations of apoA-1 and HDL were used in the NBD-cholesterol efflux assay. The medium was collected, and the cells were lysed in $0.1 \%$ Triton X-100 for at least $1 \mathrm{~h}$ at room temperature. The cell lysate and medium were each mixed with scintillation fluid and incubated for $24 \mathrm{~h}$ at room temperature, protecting the cells from light prior to scintillation counting (LS 6500 Scintillation Counter; Beckman Coulter, Brea, CA, USA). Medium radioactivity was taken for ${ }^{3} \mathrm{H}$ radioactivity determination in the scintillation counter. The cholesterol efflux rate was expressed as the percent efflux calculated by disintegrations per minute of $\left[{ }^{3} \mathrm{H}\right]$ cholesterol in medium $/\left(\left[{ }^{3} \mathrm{H}\right]\right.$ cholesterol in medium $+\left[{ }^{3} \mathrm{H}\right]$ cholesterol in cell lysates) $\mathrm{x} 100$.

To assess NBD-cholesterol efflux, macrophages were incubated in phenol red-free RPMI 1640 medium containing $5 \mu \mathrm{mol} / 1 \mathrm{NBD}$-cholesterol for $4 \mathrm{~h}$ at $37^{\circ} \mathrm{C}$. Following incubation, the cells were washed with PBS three times and were then incubated with HDL or apoA-1, as lipid acceptors. To determine the correlation, various concentrations of HDL (5, $20,50$ and $100 \mu \mathrm{g} / \mathrm{ml})$ and apoA-1 $(10,20,50$ and $100 \mu \mathrm{g} / \mathrm{ml})$ were used for the $\left[{ }^{3} \mathrm{H}\right]$-cholesterol and NBD-cholesterol efflux assays. HDL concentrations ranged between 5 and $100 \mu \mathrm{g} / \mathrm{ml}$, and apoA-1 ranged between 10 and $100 \mu \mathrm{g} / \mathrm{ml}$. Subsequently, the cells were harvested after $4 \mathrm{~h}$, and the medium and cell lysate were collected for the detection of FI. Triton X-100 $(0.1 \%)$ was used to lyse the cells in a 12 -well plate, and the cells lysate was homogenized by pipetting up and down several times. A total volume of $600 \mu \mathrm{l}$ was then aliquoted into three wells of a 96-well plate for the measurement of FI. The percentage of NBD-cholesterol efflux was calculated by dividing the FI in the medium by the sum of the FI in the medium and cell lysate. All data were from three independent experiments, each performed in triplicate.

Statistical analysis. Data were analyzed using Prism software version 5.0 (GraphPad Software, Inc., La Jolla, CA, USA). The determination of correlation was performed using Deming's regression. All data are presented as the mean \pm standard deviation. $\mathrm{P}<0.05$ was considered to indicate a statistically significant difference.

\section{Results}

NBD-cholesterol uptake and metabolism in THP-1-derived macrophages. The THP-1-derived macrophages were incubated with 0.1-10 $\mu \mathrm{M}$ 22-NBD-cholesterol and then harvested at different time-points for the determination of FI. Using a fluorescent microscope, real-time images were captured of the monolayer cells following $4 \mathrm{~h}$ incubation with NBD-cholesterol. The results revealed that NBD-cholesterol uptake from the medium occurred in a concentration- and time-dependent manner (Fig. 1C). When $0.1 \mu \mathrm{M}$ of NBD-cholesterol was used, which represents a concentration reported in several previous studies $(10,19,20)$, for up to $12 \mathrm{~h}$ of incubation, real-time imaging yielded dim fluorescent images. This finding was confirmed by quantitative FI detection, in which the FI of the cell lysate was undetectable following $12 \mathrm{~h}$ incubation. The FI increased with increasing concentrations of NBD-cholesterol, reaching the highest levels at an NBD-cholesterol concentration of $10 \mu \mathrm{M}$. Similar results were obtained in the fluorescent imaging (Fig. 1B). NBD-cholesterol uptake in the THP-1 cells also exhibited a time-dependent effect. The FI was markedly increased within the first $30 \mathrm{~min}$ of incubation and reached a plateau following incubation for $6 \mathrm{~h}$, which was observed at all concentrations, with the exception of $0.1 \mu \mathrm{M}$. Real-time imaging revealed that NBD-fluorescence was distributed equally inside the cell, but not in the nucleus.

Oxidized LDL (ox-LDL) increases NBD-cholesterol uptake by macrophages. Exogenous cholesterol is taken up by macrophages through the LDL receptor-mediated pathway, as well as by the HDL-mediated pathway (21). The present study investigated the role of ox-LDL in NBD-cholesterol uptake. The THP-1-derived macrophages were incubated with $5 \mu \mathrm{M}$ NBD-cholesterol for up to $8 \mathrm{~h}$ in the presence and absence of $50 \mu \mathrm{g} / \mathrm{ml}$ ox-LDL. The FI of the cell lysate was then measured at different time-points. The results demonstrated that ox-LDL significantly increased NBD-cholesterol uptake by the macrophages (Fig. 2).

Optimization of the NBD-cholesterol efflux assay in THP-1-derived macrophages. When using 2\% FBS to induce NBD-cholesterol efflux, the present study found that the percentage of NBD-cholesterol efflux increased in a time-dependent manner and had a marked increase within the first $30 \mathrm{~min}$, reaching a plateau after $8 \mathrm{~h}$ (Fig. 3A). Subsequently, NBD-cholesterol efflux was measured using either HDL $(50 \mu \mathrm{g} / \mathrm{ml})$ or apoA-1 $(50 \mu \mathrm{g} / \mathrm{ml})$ as lipid acceptors. The results revealed that the percentage of NBD-cholesterol efflux was increased in a similar time-dependent manner. However, when apoA-1 was used as an inducer, the percentage of efflux was markedly lower than that observed when HDL was used as an inducer (Fig. 3B and C). Based on these results, a duration of $4 \mathrm{~h}$ was set as the efflux assay duration for the subsequent NBD-cholesterol efflux assays.

In addition, the present study characterized the dose-response effect of HDL on NBD-cholesterol efflux in the THP-1 cells, using various concentrations of HDL $(5-100 \mu \mathrm{g} / \mathrm{ml})$ as a lipid acceptor. The results indicated that the percentage efflux of NBD-cholesterol increased, also in a time-dependent manner, at different concentrations of HDL (Fig. 4).

Correlation of NBD-cholesterol and $\left[{ }^{3} \mathrm{H}\right]$-cholesterol efflux in THP-1-derived macrophages. To determine the correlation between the percentage of efflux of NBD-cholesterol and $\left[{ }^{3} \mathrm{H}\right]$-cholesterol in the THP-1-derived macrophages, the present study used various concentrations of HDL and apoA-1 as lipid acceptors for the $\left[{ }^{3} \mathrm{H}\right]$-cholesterol and NBD-cholesterol efflux assays. The HDL concentrations ranged between 5 and $100 \mu \mathrm{g} /$ $\mathrm{ml}$, and the apoA-1 concentrations ranged between 10 and $100 \mu \mathrm{g} / \mathrm{ml}$ (Fig. 5). Correlation coefficiency was determined using Deming regression. The results demonstrated a significant correlation between NBD-cholesterol efflux and $\left[{ }^{3} \mathrm{H}\right]$-cholesterol efflux in the HDL- $\left(\mathrm{R}^{2}=0.876 ; \mathrm{P}<0.001\right)$ and apoA-1-mediated cholesterol efflux $\left(\mathrm{R}^{2}=0.837 ; \mathrm{P}<0.001\right)$ in the THP-1 cells, suggesting that NBD-cholesterol may be a suitable substitute for $\left[{ }^{3} \mathrm{H}\right]$-cholesterol in the cholesterol efflux assay (Fig. 5). These results also indicate a potential in vitro model for developing a 
A Cholesterol<smiles>CC(C)CCCC(C)C1CCC2C1CCC1C2CC=C2CC(O)CCC21C</smiles>

22-NBD-cholesterol<smiles>CC(C)C1CCC2C3CC=C4CC(O)CCC4(C)C3CCC12C(C)CNc1ccc([N+](=O)[O-])c2nonc12</smiles>

B
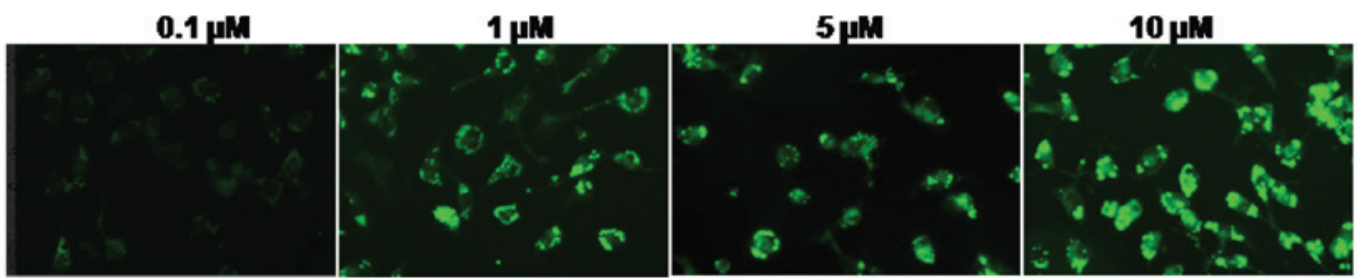

C

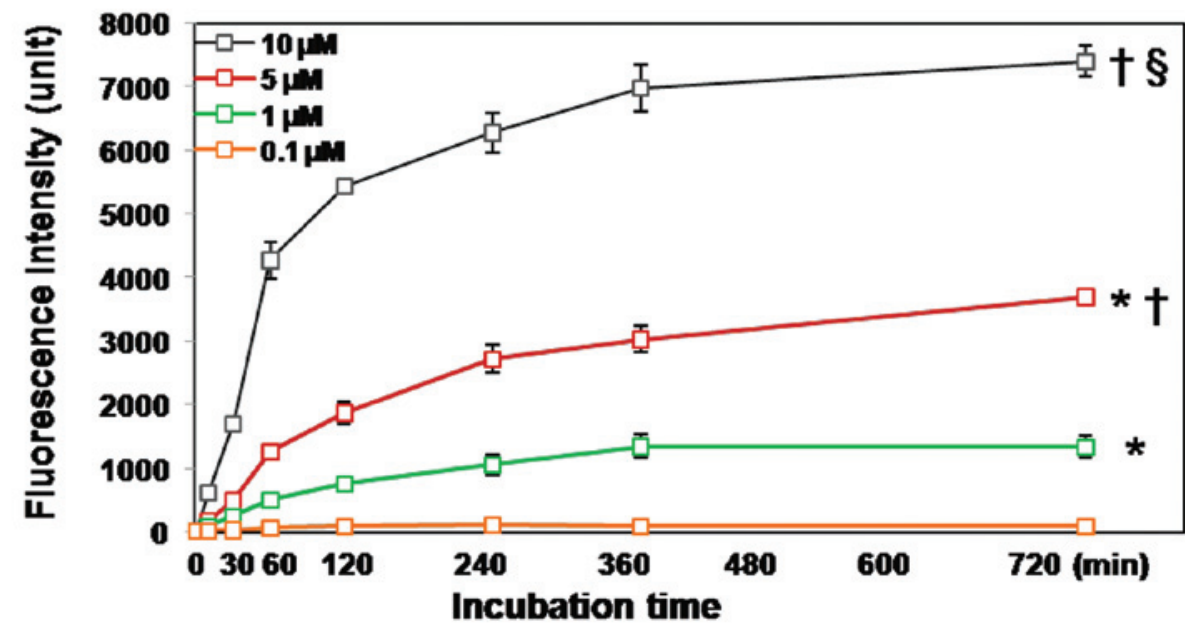

Figure 1. NBD-cholesterol uptake in THP-1 cells. (A) Molecular structures of cholesterol and 22-NBD-cholesterol. (B) Representative images of THP-1 cells treated with various concentrations of fluorescent NBD-cholesterol ranging between 0.1 and $10 \mu \mathrm{mol} / 1 \mathrm{for} 4 \mathrm{~h}$. Magnification, $\mathrm{x} 200$. (C) Quantification of the fluorescence intensity of NBD-cholesterol uptake in the THP-1 cells. THP-1 cells were treated with various concentrations of NBD-cholesterol (0.1-10 $\mu$ mol/l) and harvested at different time-points. The fluorescence intensity of the cell lysate was measured using a microplate spectrophotometer. The values are presented as the mean \pm standard deviation of triplicate wells and are representative of three independent experiments. ${ }^{\circ} \mathrm{P}<0.01$ vs. NBD $(0.1 \mu \mathrm{M})$ group; ${ }^{\dagger} \mathrm{P}<0.01$ vs. NBD $(1 \mu \mathrm{M})$ group; ${ }^{\S} \mathrm{P}<0.01$ vs. NBD $(5 \mu \mathrm{M})$ group. 22-NBD-cholesterol, 22-(7-nitrobenz-2-oxa-1, 3-diazol-4-yl-amino)-23-cholesterol.

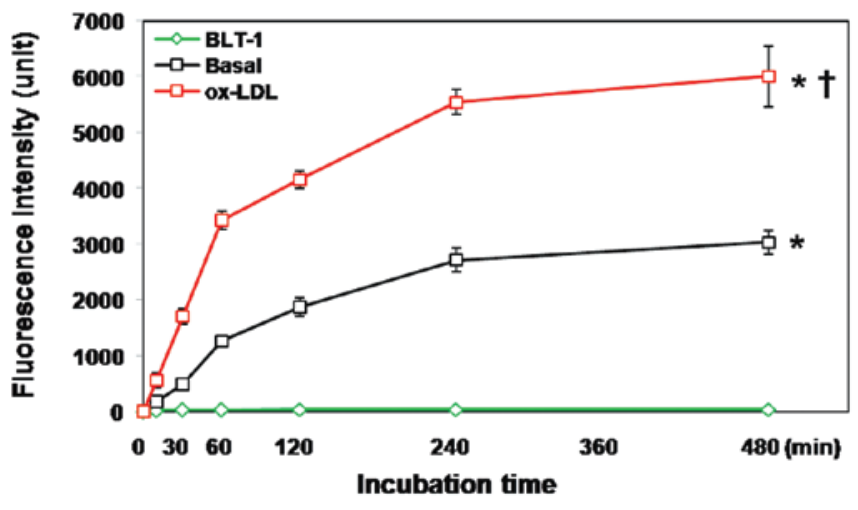

Figure 2. Effects of ox-LDL on NBD-cholesterol uptake in the THP-1 cells. THP-1 cells were treated with $50 \mu \mathrm{g} / \mathrm{ml}$ ox-LDL or medium (basal) prior to incubation with NBD-cholesterol. The cells were then labeled with NBD-cholesterol and harvested at different time-points. The fluorescence intensity was determined using a microplate spectrophotometer. The values are presented as the mean \pm standard deviation of triplicate wells and the results are representative of three independent experiments. " $\mathrm{P}<0.01$ vs. the BLT-1 group; ${ }^{\circ} \mathrm{P}<0.01$ vs. the basal group. ox-LDL, oxidized low-density lipoprotein; NBD-cholesterol, N-(7-nitrobenz-2-oxa-1,3-diazol-4-yl)amino) -23,24-bisnor-5-xholen-3 $\beta$-ol-cholesterol. sensitive, high-throughput screening method for investigating the regulation of cholesterol efflux in macrophages.

Correlation of NBD-cholesterol and $\left[{ }^{3} H\right]$-cholesterol efflux in $P B M C s$. To further assess this hypothesis, the presents study compared NBD-cholesterol with $\left[{ }^{3} \mathrm{H}\right]$-cholesterol in the efflux assay in human macrophages isolated from 13 healthy volunteers. HDL $(50 \mu \mathrm{g} / \mathrm{ml})$ and apoA-1 $(50 \mu \mathrm{g} / \mathrm{ml})$ were used as lipid acceptors for the $\left[{ }^{3} \mathrm{H}\right]$-cholesterol and NBD-cholesterol efflux assays. The results demonstrated a significant correlation between NBD-cholesterol efflux and $\left[{ }^{3} \mathrm{H}\right]$-cholesterol efflux in HDL- $\left(\mathrm{R}^{2}=0.887\right.$; $\left.\mathrm{P}<0.001\right)$ and apoA-1- $\left(\mathrm{R}^{2}=0.882\right.$; $\mathrm{P}<0.001$ ) mediated cholesterol efflux in the PBMCs (Fig. 6), which was consistent with the data obtained from the THP-1 cells.

Comparison of NBD-cholesterol efflux in THP-1-derived macrophages and PBMCs. THP-1 cells resemble human macrophages in certain biological characteristics. To validate the efficiency of these cells as macrophages in the NBD-cholesterol efflux assay, the present study further 

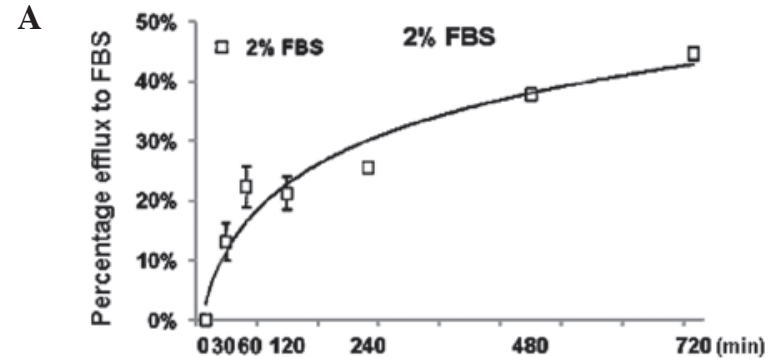

B

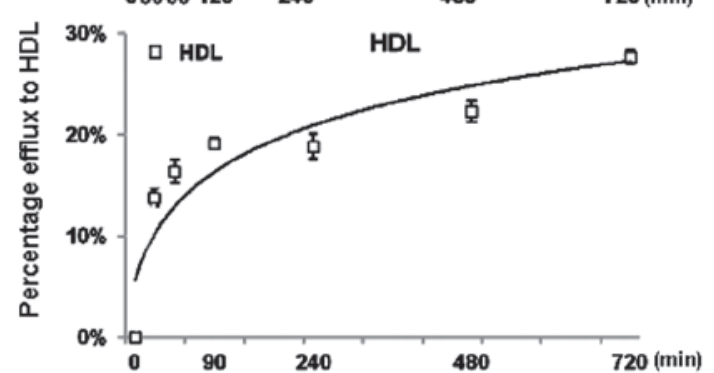

C

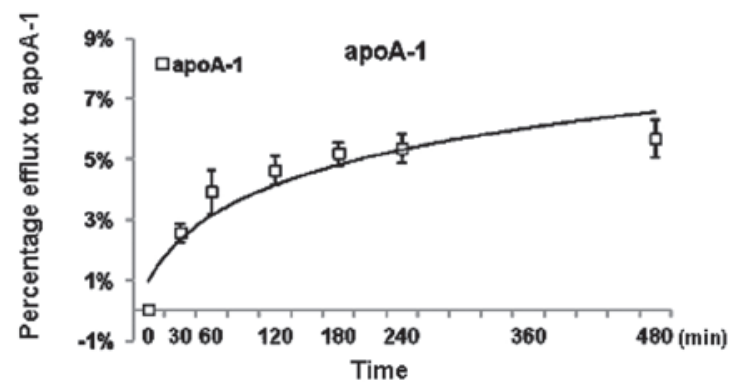

Figure 3. Optimization of the NBD-cholesterol efflux assay in THP-1 cells. Following labeling with NBD-cholesterol for $4 \mathrm{~h}$, the THP-1-derived macrophages were incubated with (A) $2 \% \mathrm{FBS}$, (B) $50 \mu \mathrm{g} / \mathrm{ml} \mathrm{HDL}$ and (C) $50 \mu \mathrm{g} / \mathrm{ml}$ apoA-1, as lipid acceptors for the cholesterol efflux assay. The percentage of NBD-cholesterol efflux was measured at the indicated times and was calculated, as described in the Materials and methods. The values are presented as the mean \pm standard deviation of triplicate wells and the data were plotted using non-linear regression. The results are representative of three independent experiments. NBD-cholesterol, N-(7-nitrobenz-2-oxa-1,3-diazol-4-yl) amino)-23,24-bisnor-5-xholen-3 $\beta$-ol-cholesterol; FBS, fetal bovine serum HDL, high-density lipoprotein; apoA-1, apolopoprotein A-1.

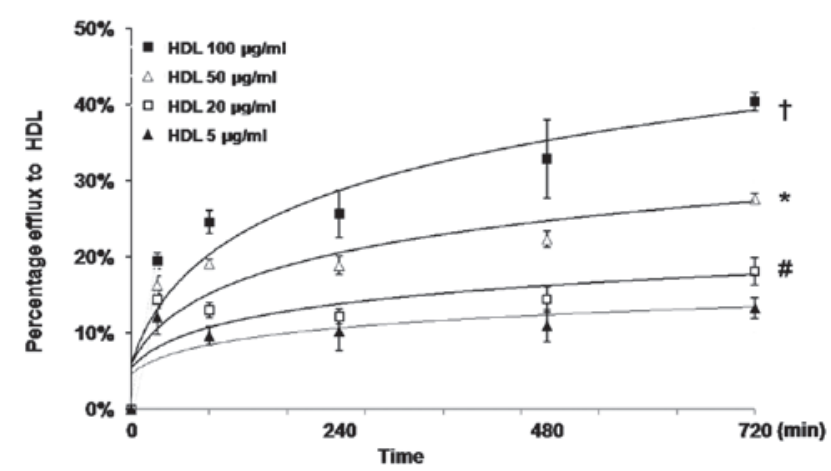

Figure 4.NBD-cholesterol efflux to different concentrations of HDL in THP-1 cells. The THP-1 cells were labeled with NBD-cholesterol for $4 \mathrm{~h}$, following which NBD-cholesterol efflux was measured using HDL as a lipid acceptor. HDL concentrations varied between 5 and $100 \mu \mathrm{g} / \mathrm{ml}$. Efflux duration was $4 \mathrm{~h}$. The fluorescence intensities of the cell lysate and medium were measured using a microplate spectrophotometer. The percentage of NBD-cholesterol efflux was calculated, as described in the Materials and methods. The values are presented as the mean \pm standard deviation of triplicate wells. Data were plotted using non-linear regression. The results are representative of at least three independent experiments. ${ }^{\prime} \mathrm{P}<0.05 \mathrm{vs}$. HDL $(5 \mu \mathrm{g} / \mathrm{ml})$ group; ${ }^{*} \mathrm{P}<0.01$ vs. HDL $(20 \mu \mathrm{g} / \mathrm{ml})$ group; ${ }^{\dagger} \mathrm{P}<0.01 \mathrm{vs}$. HDL $(50 \mu \mathrm{g} / \mathrm{ml})$ group. NBD-cholesterol, N-(7-nitrobenz-2-oxa-1,3-diazol-4-yl)amino)-23,24-bisnor-5-xholen-3 $\beta$-ol-c holesterol; HDL, high-density lipoprotein.
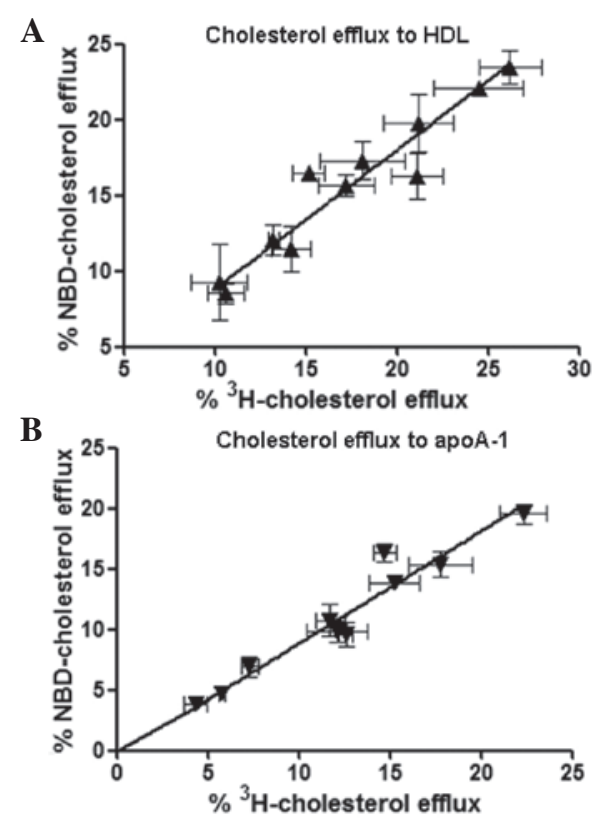

Figure 5. Correlation of NBD-cholesterol and $\left[{ }^{3} \mathrm{H}\right]$-cholesterol efflux in THP-1 cells. The THP-1 cells were labeled with either NBD-cholesterol for $4 \mathrm{~h}$ or $\left[{ }^{3} \mathrm{H}\right]$-cholesterol for $12 \mathrm{~h}$. NBD-cholesterol and $\left[{ }^{3} \mathrm{H}\right]$-cholesterol efflux were then measured using various concentrations of (A) HDL ranging between 5 and $100 \mu \mathrm{g} / \mathrm{ml}$ and (B) apoA-1 ranging between 10 and $100 \mu \mathrm{g} / \mathrm{ml}$, as lipid acceptors. Efflux duration was $4 \mathrm{~h}$. Correlation efficiency was determined using Deming's regression $(\mathrm{P}<0.001)$. NBD-cholesterol, $\mathrm{N}$-(7-nitrobenz-2-oxa-1,3-diazol-4-yl)amino)-23,24-bisnor-5-xholen-3 3 -ol-c holesterol; HDL, high-density lipoprotein; apoA-1, apolopoprotein A-1.
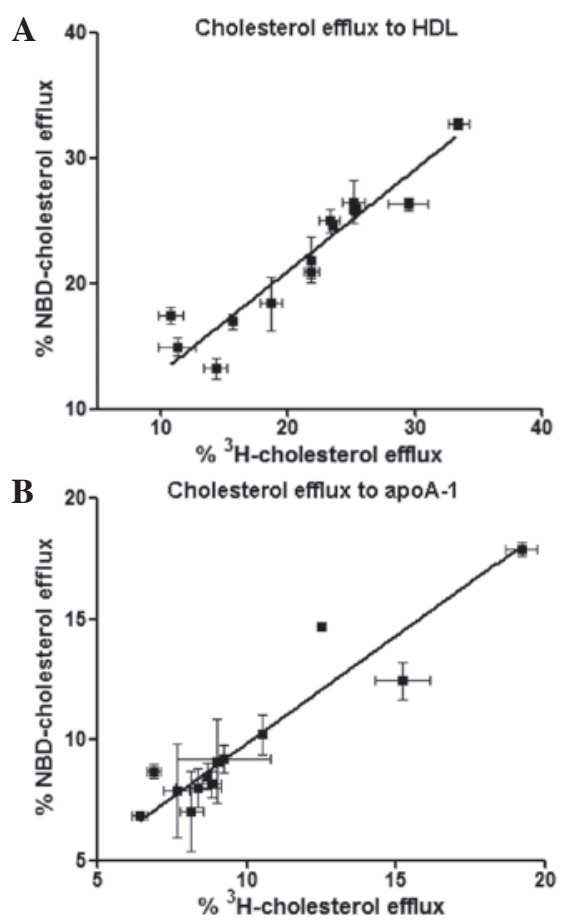

Figure 6. Correlation of NBD-cholesterol and $\left[{ }^{3} \mathrm{H}\right]$-cholesterol efflux in PBMCs. Following labeling PBMCs with either NBD-cholesterol for $4 \mathrm{~h}$ or $\left[{ }^{3} \mathrm{H}\right]$-cholesterol for $12 \mathrm{~h}, \mathrm{NBD}$-cholesterol and $\left[{ }^{3} \mathrm{H}\right]$-cholesterol efflux were measured using (A) $50 \mu \mathrm{g} / \mathrm{ml} \mathrm{HDL}$ or (B) $50 \mu \mathrm{g} / \mathrm{ml}$ apoA-1 as lipid acceptors. Efflux duration was $4 \mathrm{~h}$. The fluorescence intensity of the cell lysate or medium was measured using a microplate spectrophotometer. Correlation efficiency was determined using Deming's regression $(\mathrm{P}<0.001)$. The results are representative of three independent experiments, each performed in triplicate. PBMCs, peripheral blood mononuclear cells; NBD-cholesterol, $\mathrm{N}$-(7-nitrobenz-2-oxa-1,3-diazol-4-yl)amino)-23,24-bisnor-5-xholen-3 $\beta$-ol-c holesterol; HDL, high-density lipoprotein; apoA-1, apolopoprotein A-1. 
A

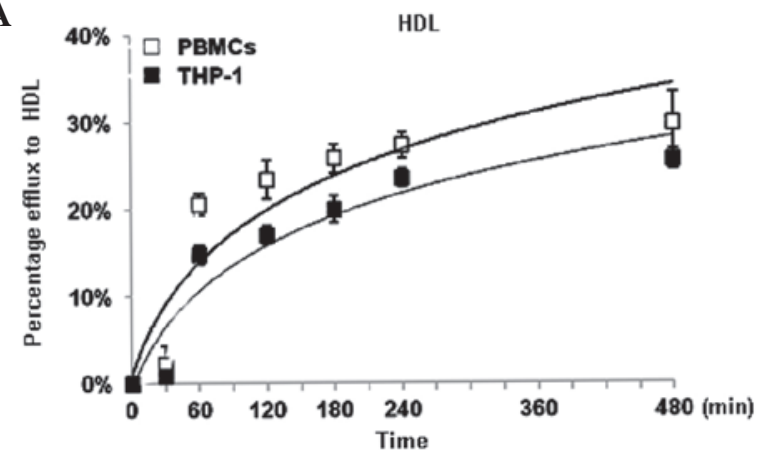

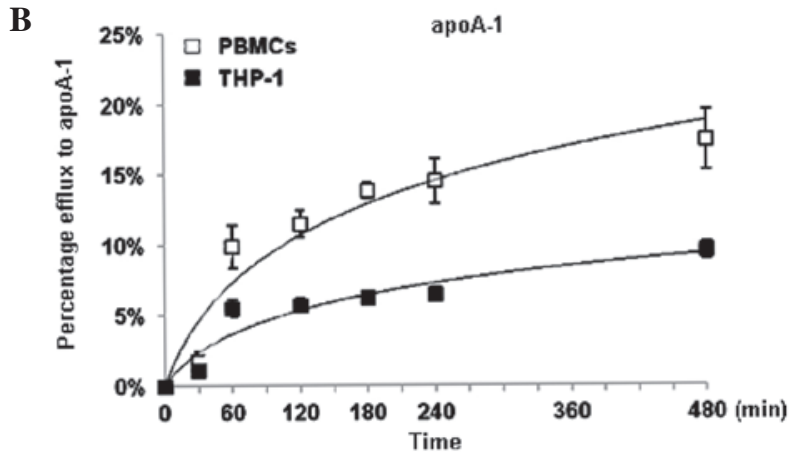

Figure 7. Comparison of NBD-cholesterol efflux between different cells. THP-1 cells were differentiated into macrophages by treatment with 100 ng/ml phorbol myristate acetate for $72 \mathrm{~h}$. PBMCs were differentiated into macrophages, by treatment in serum-free RPMI 1640 medium containing 25 mmol/1 HEPES and $10 \mathrm{ng} / \mathrm{ml}$ human macrophage colony stimulating factor. The cells were labeled with NBD-cholesterol for $4 \mathrm{~h}$. NBD-cholesterol efflux was measured in the THP-1 cells or PBMCs using (A) $50 \mu \mathrm{g} / \mathrm{ml}$ HDL or (B) $50 \mu \mathrm{g} / \mathrm{ml}$ apoA-1 as lipid acceptors. Efflux duration was 4 h. The values are presented as the mean \pm standard deviation. Data were plotted using non-linear regression. The results are representative of three independent experiments, each performed in triplicate. PBMCs, peripheral blood mononuclear cells; NBD-cholesterol, N-(7-nitrobenz-2-oxa-1,3-diazol-4-yl)amino)-23,24-bisnor-5-xholen-3 $\beta$-ol-cholest erol; HDL, high-density lipoprotein; apoA-1, apolopoprotein A-1.

compared the THP-1 cells with the PBMCs isolated from volunteers. HDL and apoA-1 were used as lipid acceptors. The percentages of NBD-cholesterol efflux in the THP-1 cells and PBMCs were markedly increased within the first hour, and continuously increased between 1 and $4 \mathrm{~h}$ (Fig. 7). The percentage of efflux of NBD-cholesterol reached a peak at $8 \mathrm{~h}$ with either HDL or apoA-1 as an inducer. Although the percentage of NBD-cholesterol efflux in the THP-1 cells appeared lower than that in the PBMCs, NBD-cholesterol efflux in the THP-1 cells exhibited a similar trend to that in the PBMCs, suggesting that THP-1 cells may be a suitable alternative model in this NBD-cholesterol efflux assay (Fig. 7).

\section{Discussion}

Fluorescent cholesterol analogs, which can closely mimic the properties of natural cholesterol, whilst permitting detection by microscopic techniques, have been widely used to investigate the intracellular distribution and membrane organization of cholesterol. These fluorescent cholesterol analogs have also demonstrated significant potential as substitutes for traditional radioisotope-labeled cholesterol for investigating lipid trafficking, including cholesterol efflux $(7,22)$. Cholesterol efflux may prevent the development of atherosclerosis by reducing the accumulation of cholesterol in the artery wall $(2,23)$ and is a key step in RCT. Understanding the regulation of cholesterol efflux can be useful therapeutically and merits in-depth investigation. Previously, Zhang et al developed fluorescent cholesterol bearing the Pennsylvania Green fluorophore for use as a molecular probe for the cholesterol efflux assay. This fluorescent sterol exhibits good correlation with radioisotope-labeled cholesterol in the efflux assay in THP-1 cells (24). Sankaranarayanan et al demonstrated that another fluorescent sterol, bearing a BODIPY fluorophore, can be used as a sensitive probe in the cholesterol efflux assay in J774 mouse macrophages (11). NBD-cholesterol is a commercially available and widely used fluorescent analog of cholesterol, which may be used in cholesterol efflux assays (25-27). Frolov et al used 22-NBD-cholesterol in an investigation of HDL-mediated cholesterol efflux in mouse L-cells (21). Storey et al also used this fluorescent sterol in an investigation of HDL-mediated cholesterol efflux in cultured primary mouse hepatocytes (10). Although these studies demonstrated the potential application of NBD-cholesterol, there remains a lack of data regarding NBD-cholesterol uptake and efflux in THP-1 cells, which is a more physiologically relevant human cell line that exhibits macrophage-like characteristics. In the present study, the cholesterol efflux assay was characterized and optimized using fluorescent NBD-cholesterol in THP-1-derived macrophages.

The initial step of a cholesterol efflux assay is to pre-incubate cells with NBD-cholesterol, which is a prerequisite for the assay (22). Therefore, the present study first characterized NBD-cholesterol uptake in the THP-1-derived macrophages and optimized the experimental conditions. The cells were incubated with various concentrations of NBD-cholesterol, and the cellular FI was measured at different time-points. The results revealed that NBD-cholesterol uptake in the THP-1 cells was increased over time and reached a plateau after $4 \mathrm{~h}$ incubation, suggesting that intracellular NBD-cholesterol metabolism may reach equilibrium over time. The results also demonstrated that pre-incubation of the cells with $5 \mu \mathrm{M}$ NBD-cholesterol resulted in optimal outcomes in fluorescence microscopy and scintillation counting. Thus, the optimized conditions for cellular NBD-cholesterol uptake were established for the subsequent experiments. Previously, Storey et al investigated cholesterol uptake in cultured primary mouse hepatocytes using $0.1 \mu \mathrm{M}$ NBD-cholesterol (10), which was a lower level than that used in the present study. In another study, Portioli Silva et al analyzed cholesterol incorporation in rat macrophages using $2 \mu \mathrm{M}$ NBD-cholesterol, which was close to the concentration used in our study (28). Notably, the present study used ethanol as a specific solvent for NBD-cholesterol solubilization, which was also used in the study by Portioli Silva et al, but not in the study by Storey et al. The use of a solvent is important for the fluorescence quantum yield and extinction coefficient, and may be the cause of the difference between the results of the studies (8).

The present study also demonstrated that by treating the cells with ox-LDL, NBD-cholesterol uptake in the THP-1 cells was significantly increased. Previous studies have reported that 
ox-LDL is a specific ligand for peroxisome proliferator-activated receptors and, upon binding, may increase its target gene expression and regulate lipid homeostasis $(29,30)$. The results of the present study demonstrated that NBD-cholesterol absorption in macrophages was increased by ox-LDL treatment, which was similar to native cholesterol.

Subsequently, the present study investigated whether NBD-cholesterol efflux was well-correlated with $\left[{ }^{3} \mathrm{H}\right]$-labeled cholesterol efflux in the THP-1-derived macrophages. $\left[{ }^{3} \mathrm{H}\right]$ radioisotope-labeling is a classical labeling method for investigating intracellular cholesterol trafficking and metabolism due to its sensitivity and specificity. In the present study, $\left[{ }^{3} \mathrm{H}\right]$-cholesterol efflux and fluorescent NBD-cholesterol efflux were measured in the THP-1 cells using various concentrations of HDL or apoA-1 as lipid acceptors. The data revealed a significant correlation between NBD-cholesterol efflux and $\left[{ }^{3} \mathrm{H}\right]$-cholesterol efflux in the THP-1 cells. In addition, NBD-cholesterol efflux and $\left[{ }^{3} \mathrm{H}\right]$-cholesterol effluxwere examined in PBMCs isolated from healthy volunteers. A significant correlation between NBD-cholesterol efflux and $\left[{ }^{3} \mathrm{H}\right]$-cholesterol efflux was also observed in the PBMCs.

Cellular cholesterol transportation to extracellular acceptors either occurs through aqueous diffusion or is mediated by the ABCA1 or ABCG1 transmembrane proteins, which are essential in the process (31-33). Previous studies have revealed that cellular cholesterol is transported to either lipid-depleted apoA-1 through the ABCA1-mediated pathway or HDL through the ABCG1-mediated pathway (34-36). Therefore, the present study used apoA-1 and HDL as extracellular acceptors for evaluating cellular cholesterol efflux. The resulting data demonstrated that the percentage of NBD-cholesterol efflux was significantly correlated to that of $\left[{ }^{3} \mathrm{H}\right]$-cholesterol in the different cholesterol efflux pathways in macrophages. Thus, these findings support NBD-cholesterol as a sensitive and specific probe, which may be used efficiently for cholesterol efflux measurement. Several other studies have reported that fluorescent BODIPY-cholesterol and Pennsylvania Green-cholesterol can also be used in cholesterol efflux assay as substitutes for $\left[{ }^{3} \mathrm{H}\right]$-cholesterol $(11,24)$. Further investigation to compare different fluorescent analogs in parallel with $\left[{ }^{3} \mathrm{H}\right]$-cholesterol may assist in determining which fluorescent analog can mimic native cholesterol efflux most closely in the assay.

The THP-1 cells used in the present study are a human monocytic leukemia cell line, which can be differentiated into macrophage-like cells. Therefore, THP-1 cells were used as an in vitro model relevant to human macrophages for the NBD-cholesterol efflux assay. It is important to determine whether NBD-cholesterol metabolism and efflux in THP-1 cells is similar to that in PBMCs. In the present study, NBD-cholesterol efflux in THP-1 cells was compared with that in PBMC. NBD-cholesterol efflux in the THP-1 cells exhibited a similar trend to that in PBMCs, suggesting that THP-1 is a suitable model for assessing NBD-cholesterol efflux in macrophages. By contrast, with regard to the lower percentage of NBD-cholesterol efflux observed in THP-1 cells than in the PBMCs, it is possible that the expression levels of lipid transporters, including ABCA1 may be lower in THP-1 cells than in PBMCs, which may be responsible for a lower efflux percentage. Further investigation on the comparison between THP-1 cells and PBMCs, in terms of the expression and function of ABCA1 amay answer this important question.

In conclusion, the present study demonstrated a simpler procedure with a similar efficiency for performing fluorescent NBD-cholesterol efflux assays, compared with $\left[{ }^{3} \mathrm{H}\right]$ radioisotope-labeled cholesterol. The results may assist in the development of a rapid sensitive high-throughput screening assay for investigating macrophage cholesterol efflux.

\section{Acknowledgements}

The present study was supported by a grant from Pfizer Inc. (New York, NY, USA; grant. no. WS554487).

\section{References}

1. Yusuf S, Reddy S, Ounpuu S and Anand S: Global burden of cardiovascular diseases: Part I: General considerations, the epidemiologic transition, risk factors and impact of urbanization. Circulation 104: 2746-2753, 2001.

2. Ross R: Atherosclerosis-an inflammatory disease. N Engl J Med 340: 115-126, 1999.

3. Fielding CJ and Fielding PE: Cellular cholesterol efflux. Biochim Biophys Acta 1533: 175-189, 2001.

4. Tall AR, Costet P and Wang N: Regulation and mechanisms of macrophage cholesterol efflux. J Clin Invest 110: 899-904, 2002.

5. Cuchel M and Rader DJ: Macrophage reverse cholesterol transport: Key to the regression of atherosclerosis? Circulation 113: 2548-2555, 2006.

6. Khera AV, Cuchel M, de la Llera-Moya M, Rodrigues A, Burke MF, Jafri K, French BC, Phillips JA, Mucksavage ML, Wilensky RL, et al: Cholesterol efflux capacity, high-density lipoprotein function and atherosclerosis. N Engl J Med 364: 127-135, 2011.

7. Zhang J, Cai S, Peterson BR, Kris-Etherton PM and Heuvel JP: Development of a cell-based, high-throughput screening assay for cholesterol efflux using a fluorescent mimic of cholesterol. Assay Drug Dev Technol 9: 136-146, 2011.

8. Wustner D: Fluorescent sterols as tools in membrane biophysics and cell biology. Chem Phys Lipids 146: 1-25, 2007.

9. Ramirez DM, Ogilvie WW and Johnston LJ: NBD-cholesterol probes to track cholesterol distribution in model membranes. Biochim Biophys Acta 1798: 558-568, 2010.

10. Storey SM, Atshaves BP, McIntosh AL, Landrock KK, Martin GG, Huang H, Ross Payne H, Johnson JD, Macfarlane RD, Kier AB and Schroeder F: Effect of sterol carrier protein-2 gene ablation on HDL-mediated cholesterol efflux from cultured primary mouse hepatocytes. Am J Physiol Gastrointest Liver Physiol 299: G244-G254, 2010

11. Sankaranarayanan S, Kellner-Weibel G, de la Llera-Moya M, Phillips MC, Asztalos BF, Bittman R and Rothblat GH: A sensitive assay for ABCA1-mediated cholesterol efflux using BODIPY-cholesterol. J Lipid Res 52: 2332-2340, 2011.

12. Tsuchiya S, Yamabe M, Yamaguchi Y, Kobayashi Y, Konno T and Tada K: Establishment and characterization of a human acute monocytic leukemia cell line (THP-1). Int J Cancer 26: 171-176, 1980.

13. Tsuchiya S, Kobayashi Y, Goto Y, Okumura H, Nakae S, Konno T and Tada K: Induction of maturation in cultured human monocytic leukemia cells by a phorbol diester. Cancer Res 42: 1530-1536, 1982

14. Liu XH, Xiao J, Mo ZC, Yin K, Jiang J, Cui LB, Tan CZ, Tang YL, Liao DF and Tang CK: Contribution of D4-F to ABCA1 expression and cholesterol efflux in THP-1 macrophage-derived foam cells. J Cardiovasc Pharmacol 56: 309-319, 2010.

15. Larrede S, Quinn CM, Jessup W, Frisdal E, Olivier M, Hsieh V, Kim MJ, Van Eck M, Couvert P, Carrie A, et al: Stimulation of cholesterol efflux by LXR agonists in cholesterol-loaded human macrophages is ABCA1-dependent but ABCG1-independent. Arterioscler Thromb Vasc Biol 29: 1930-1936, 2009.

16. Patel S, Drew BG, Nakhla S, Duffy SJ, Murphy AJ, Barter PJ, Rye KA, Chin-Dusting J, Hoang A, Sviridov D, et al: Reconstituted high-density lipoprotein increases plasma high-density lipoprotein anti-inflammatory properties and cholesterol efflux capacity in patients with type 2 diabetes. J Am Coll Cardiol 53: 962-971, 2009. 
17. de Almeida MC, Silva AC, Barral A and Barral Netto M: A simple method for human peripheral blood monocyte isolation. Mem Inst Oswaldo Cruz 95: 221-223, 2000.

18. Xu Y, Wang W, Zhang L, Qi LP, Li LY, Chen LF, Fang Q, Dang AM and Yan XW: A polymorphism in the ABCG1 promoter is functionally associated with coronary artery disease in a Chinese Han population. Atherosclerosis 219: 648-654, 2011.

19. Fan J, Rone MB and Papadopoulos V: Translocator protein 2 is involved in cholesterol redistribution during erythropoiesis. J Biol Chem 284: 30484-30497, 2009.

20. Sasaki $\mathrm{H}$ and White $\mathrm{SH}$ : A novel fluorescent probe that senses the physical state of lipid bilayers. Biophys J 96: 4631-4641, 2009.

21. Frolov A, Petrescu A, Atshaves BP, So PT, Gratton E, Serrero G and Schroeder F: High density lipoprotein-mediated cholestero uptake and targeting to lipid droplets in intact L-cell fibroblasts. A single- and multiphoton fluorescence approach. J Biol Chem 275: 12769-12780, 2000.

22. Sengupta B, Narasimhulu CA and Parthasarathy S: Novel technique for generating macrophage foam cells for in vitro reverse cholesterol transport studies. J Lipid Res 54: 3358-3372, 2013.

23. Ohashi R, Mu H, Wang X, Yao Q and Chen C: Reverse cholesterol transport and cholesterol efflux in atherosclerosis. QJM 98: 845-856, 2005

24. Zhang J, Cai S, Peterson BR, Kris-Etherton PM and Heuvel JP Development of a cell-based, high-throughput screening assay for cholesterol efflux using a fluorescent mimic of cholesterol. Assay Drug Dev Technol 9: 136-146, 2011.

25. Ohashi M, Murata M and Ohnishi S: A novel fluorescence method to monitor the lysosomal disintegration of low density lipoprotein. Eur J Cell Biol 59: 116-126, 1992.

26. Adams MR, Konaniah E, Cash JG and Hui DY: Use of NBD-cholesterol to identify a minor but NPC1L1-independent cholesterol absorption pathway in mouse intestine. Am J Physiol Gastrointest Liver Physiol 300: G164-G169, 2011.

27. Takahashi M, Murate M, Fukuda M, Sato SB, Ohta A and Kobayashi T: Cholesterol controls lipid endocytosis through Rab11. Mol Biol Cell 18: 2667-2677, 2007.
28. Portioli Silva EP, Peres CM, Roberto Mendonca J and Curi R: NBD-cholesterol incorporation by rat macrophages and lymphocytes: A process dependent on the activation state of the cells. Cell Biochem Funct 22: 23-28, 2004.

29. Nagy L, Tontonoz P, Alvarez JG, Chen H and Evans RM Oxidized LDL regulates macrophage gene expression through ligand activation of PPARgamma. Cell 93: 229-240, 1998.

30. Boullier A, Bird DA, Chang MK, Dennis EA, Friedman P, Gillotre-Taylor K, Hörkkö S, Palinski W, Quehenberger O, Shaw $\mathrm{P}$, et al: Scavenger receptors, oxidized LDL and atherosclerosis. Ann N Y Acad Sci 947: 214-222; discussion 222-223, 2001.

31. Yvan-Charvet L, Ranalletta M, Wang N, Han S, Terasaka N, Li R, Welch C and Tall AR: Combined deficiency of ABCA1 and ABCG1 promotes foam cell accumulation and accelerates atherosclerosis in mice. J Clin Invest 117: 3900-3908, 2007.

32. Adorni MP, Zimetti F, Billheimer JT, Wang N, Rader DJ, Phillips MC and Rothblat GH: The roles of different pathways in the release of cholesterol from macrophages. J Lipid Res 48: 2453-2462, 2007.

33. Wang X, Collins HL, Ranalletta M, Fuki IV, Billheimer JT, Rothblat GH, Tall AR and Rader DJ: Macrophage ABCA1 and ABCG1, but not SR-BI, promote macrophage reverse cholesterol transport in vivo. J Clin Invest 117: 2216-2224, 2007.

34. Wang N, Lan D, Chen W, Matsuura F and Tall AR: ATP-binding cassette transporters G1 and G4 mediate cellular cholesterol efflux to high-density lipoproteins. Proc Natl Acad Sci USA 101: 9774-9779, 2004

35. Tall AR, Yvan-Charvet L, Terasaka N, Pagler T and Wang N: HDL, ABC transporters and cholesterol efflux: Implications for the treatment of atherosclerosis. Cell Metab 7: 365-375, 2008.

36. Yvan-Charvet L, Wang N and Tall AR: Role Of HDL, ABCA1 and ABCG1 transporters in cholesterol efflux and immune responses. Arterioscler Thromb Vasc Biol 30: 139-143, 2010. 